\title{
Microchimica Acta special issue MIP2012 on Molecularly imprinted polymers - science and technology
}

\author{
Karsten Haupt
}

Received: 27 September 2013 / Accepted: 27 September 2013 /Published online: 10 October 2013

(C) Springer-Verlag Wien 2013

\section{Preface}

Like a key in a lock, antibodies perfectly fit their target antigens and are able to recognize and bind them with high affinity and selectivity. They have, therefore, found numerous applications in medicine, both for diagnostics and treatment, and in biotechnology. However, antibodies have some limitations for these applications - they are unstable out of their native environment, may be degraded by proteases, and tend to be difficult to integrate into standard industrial fabrication processes. Being able to obtain such structures synthetically, to create tailor-made receptors for a given molecular target using a biomimicry approach, is therefore of great interest. One surprisingly simple way of generating 'plastic antibodies' is through the molecular imprinting of synthetic polymers.

Molecular imprinting is a process where a polymer is synthesized in the presence of a molecular template, a process that generates cavities within the polymer network that are complementary to the template molecule in size, shape, and position of functional groups, the cross-linked polymer matrix preserving their three-dimensional structure. These molecularly imprinted polymers (MIPs) now possess two of the most important features of biological antibodies - the ability to recognize and bind specific target molecules. There are a number of application areas that have been identified for MIPs, all based on their specific recognition capability: affinity separation, chemical sensors, pseudo-immunoassays, directed synthesis and enzyme-like catalysis, and biomedical applications like drug delivery and medical treatment.

\section{K. Haupt $(\bowtie)$}

CNRS Institute for Enzyme and Cell Engineering,

Compiègne University of Technology,

60205 Compiègne cedex, France

e-mail: karsten.haupt@utc.fr
In August 2012, the 7th International Conference on Molecularly Imprinted Polymers (MIP2012) took place in Paris, France, within a biannual series of MIP conferences. Part of the activities were organized on the campus of the Ecole Supérieure de Physique et de Chimie Industrielles de la Ville de Paris, the very place where Marie Curie conducted her pioneering research on radioactivity and discovered the elements radium and polonium. About 200 scientists from over 30 countries, including many young scientists, attended the conference. It nicely coincided with the 40th anniversary of the first paper that has initiated the modern area of Molecular Imprinting.

It was indeed very exciting to see the strong and broad development of the MIP area during the past years, which was reflected by the scientific programme of the conference. When these molecularly imprinted polymers were first described in the 70s and early 1980s by Wulff, Mosbach and Shea, they were merely used as specific separation materials, for example for the chromatographic separation of enantiomers. It was not until 1993 and a seminal paper by Mosbach in Nature that the great potential of MIPs as synthetic antibody mimics was recognized. This resulted in a nearly exponential increase in the number of publications in the area, with several hundreds per annum over the recent years. It should be mentioned here that the leading role of the two 'fathers' of molecular imprinting, Günter Wulff and Klaus Mosbach, has been put forward at MIP2012 in form of an honorary ceremony, where they received a special distinction. A review article by Günter Wulff, which is included in this special issue, describes nicely the historical and more recent developments in Molecular Imprinting, with special emphasis on enzyme mimics. A review by Klaus Mosbach on the beginnings and on the future of the area will be included early in 2014 in an issue of Microchimica Acta .

So, where do we go from here? From a materials point of view, there is still much room for improvement of MIPs. Indeed, during the past 10 years, the development in the area has taken a few different directions aiming at making these 
improvements possible. For example, it has been suggested to apply controlled polymerization techniques for MIP synthesis, in order to improve their inner morphology. Another trend is the combination of molecular imprinting and nanostructures, yielding materials with interesting additional properties, like photonic crystals and holograms. An important development is the systematic decrease of particle size, resulting in nanogels with sizes in the lower nanometer range, which seems to convey to MIPs properties close to those of biological antibodies. These include for example solubility, the presence of only one binding site per particle, and a homogeneous binding site affinity distribution. This is put forward for example by the groups of Shea, Piletsky, and by our group. Resmini has shown that these particles, when imprinted with a transition state analog, can be efficient enzyme mimics. The development of MIPs that can recognize proteins has also been a long time dream of many researchers work- ing in the area, which now seems to come true, an example being MIPs that very specifically recognize peptide epitopes of proteins.

Overall, it appears that MIPs will finally find their own applications, rather than trying to do what antibodies already can do, and better. Industry is currently evaluating potential applications and opportunities for MIPs. Thereby, companies need materials that selectively recognize their targets not in pure solvents in the 'imprinter's lab', but in real-world applications, for example in biological fluids or in environmental waters. Criteria like the ready integration of molecular imprinting within existing industrial fabrication processes, yields, cost, and the competitiveness of MIPs with existing affinity materials are also important. All this can trigger new developments that will lead to considerable improvements of MIP materials, but also leads to new challenges which the community is looking forward to address. 